\title{
A simple Fibonacci weighted scoring system to rate examinations of children with respiratory illness
}

\begin{abstract}
A thorough examination of the head and neck is necessary to establish a diagnosis in pyrexial children, and for the exclusion of rare, but not to be missed conditions, such as Lymphoma and EBV. Scoring systems are a key tool in providing feedback to clinicians on how well these are carried out, and tracking their improvements.

This audit looked at how well children with suspected respiratory illness were examined and how this was improved with such a tool. Feverish children over the age of 6 months were tracked: by whom they saw and how well they were examined. The quality of examination was stratified under a 3-teir standard (Gold, Silver and Bronze) with the "Gold" examinations including correct documentation of the state of ears, throat and lymph nodes, with Silver and Bronze allocated to standards approaching this.
\end{abstract}

This Fennobacci-weighted scoring system was employed to average a score achieved by each grade over 21-month periods. Clinicians had re-education on the clinical exams in the interim. Junior doctors improved scores by $25 \%$, nurse practitioners by $112 \%$ and more senior grades by $6 \%$. The scoring system proved a motivational feedback mechanism across teams.

Revalidation and confidence-building are required for doctors in training to achieve a high consistency in clinical examination, and novel scoring systems can be helpful in achieving this.

Keywords: otolaryngology, paediatrics, clinical examination, fever, COVID 19
Volume 9 Issue 3 - 2020

Flynn MF, Blacklock CT
Royal Alexandra Hospital, United Kingdom

Correspondence: Matthew Flynn,Victoria Hospital, Victoria Hospital, Hayfield Road, Kirkcaldy, KY2 5AH, UK, +447786018918, Email m.flynn5@nhs.net https://orcid.org/0000-0002-0499-9777

Received: May 14, 2020 | Published: June II, 2020
Abbreviations: SHO, senior house officer; ANP, advanced nurse practitioner; FA, first assessor; ENT, ear nose and throat

\section{Introduction}

Respiratory infections of the head and neck are a common presentation in the paediatric population, but, being routine, present an opportunity for serious pathology to be missed. Epstein-Barr Virus, Kawasakis' Disease and Lymphoma, for instance, can present with signs not dissimilar to the common cold. A thorough examination of the head and neck is necessary to establish a diagnosis in pyrexial children, and for the exclusion of these rarer, but not to be missed, conditions. ${ }^{\mathrm{i}, \mathrm{ii}, \mathrm{iii}}$ Such in depth clinical examinations are not often employed outwith the specialty of otolaryngology, but paediatrics is an area where they are well employed..$^{\text {iv }}$ In light of the coronavirus pandemic of 2020, the effective triage and discharge of children with respiratory infections from hospital has heightened relevance. ${ }^{\mathrm{v}, \mathrm{vi}}$

This project established a feedback mechanism for clinicians in training, and examined whether simple re-education of medical staff suffices to elicit a detectable improvement in practice, aiding safe discharge of these children. The audit was conducted in a Paediatric Assessment Unit in a District General Hospital receiving referrals from primary care, from where patients were to be either admitted or discharged based on this assessment.

Scoring systems have been used effectively to stratify clinical competencies across grades of clinicians. ${ }^{\text {viiviii }}$ A Fenobacci weighted points system, such as that used in the Olympic games to declare overall leaders across diverse teams, was used to asses overall performance of each clinician team. ${ }^{\text {ix }}$ This led to development of a Gold/Silver/Bronze rating, which was hoped to be motivational.

\section{Materials and methods}

\section{Case note review}

Case note records were obtained for 2 groups of patients (43 and 36 respectively) in two cycles of analysis, by Foundation Year 2 Doctors and GP trainees (collectively SHO's), Advanced Nurse Practitioners (ANP's) and Paediatric Specialty Trainees and Staff Grades (Registrars). Included were children who were older than 6 months, those presenting to the acute paediatric assessment unit (accepting ages $0-15$ ), and temperature $>37.5^{\circ} \mathrm{C}$ or "Pyrexia," "Fever," "Hot" or "[High] Temperature" recorded on either nursing or medical admission notes. Excluded were examinations by medical students or those conducted in the emergency department.

\section{Fenobacci scoring system}

The results were collated by clinician group they saw and how well they were examined. A 1:2:3 $(a+b=c)$ *scoring system was employed: full documentation of the ear, throat and neck was awarded 3 points, a focussed examination of two of these systems qualified 2, and any other documented attempt of ENT examination falling short of these, was awarded 1 (Table 1).

"In Fibonacci sequences, a higher number is the sum of the two preceding, ensuring the 'gold standard' examinations contributed to scoring as much as two poorer examinations combined [IX] 
Table I Necessary documented examination for each score

\begin{tabular}{llll}
\hline Necessary documentation & Bronze & Silver & Gold \\
\hline Documented neck examination & $X$ & $X$ & $X$ \\
\hline "ENT:NAD"* & $X$ & $X$ \\
\hline Focussed documented examination of ears and throat & $X$ & \\
\hline Any documentation of ENT examination below above standards & $X$ & \\
\hline No documentation & $\times$ & \\
\hline
\end{tabular}

*Nasal examination was not deemed an essential component and was seldom otherwise documented

ENT, Ear, nose and throat; NAD, Nothing abnormal detected

\section{Collation of scores by clinician grade}

The standards of the examinations being thus identified, a mean of the maximum score of 3 would be applied to each subgroup of clinicians. The process was repeated after an interval of a month, with the aim of demonstrating an augmentation of scores.

\section{Action taken}

A departmental presentation was given on the first round of results, highlighting the clinical relevance of the ENT examination, and clinicians were invited to participate in an online survey, summarising attitudes towards what was considered effective. The national guidelines for assessment of feverish children were signposted.

\section{Results (Graph I-3)}

During the first cycle the SHO's (Regs, ANP's) completed a total of $23(16,2)$ examinations which grew to $29(12,7)$. As a cohort SHO's demonstrated improvement in scores towards the gold standard, with an increase in net score of 2.17 to 2.34 in response to retraining. Registrar level doctors showed no overall improvement, with their decreased rate of attaining gold-level examinations being proportional to the decrease in bronze level. From the relatively small numbers of ANP's sampled there was a noticeable improvement in scores from 1.00 to 1.57 .

\begin{tabular}{lllllll}
\hline \multirow{2}{*}{ Number of Examinations per category } & \multicolumn{2}{l}{ SHO } & & Reg & \multicolumn{2}{l}{ ANP } \\
\cline { 2 - 7 } & Cycle I & Cycle 2 & Cycle I & Cycle 2 & Cycle I & Cycle 2 \\
\hline Bronze = Ipt & 4 & 5 & 4 & 2 & 2 & 3 \\
\hline Silver = 2pts & II & 9 & 8 & 8 & 0 & 4 \\
\hline Gold = 3pts & 8 & 15 & 4 & 2 & 0 & 0 \\
\hline Average fenobacci weighted score & 2.17 & 2.34 & 2 & 2 & 1 & 1.57 \\
\hline
\end{tabular}

$\mathrm{SHO}$, senior house officer; Reg, registrar staff grade; ANP, advanced nurse practitioner

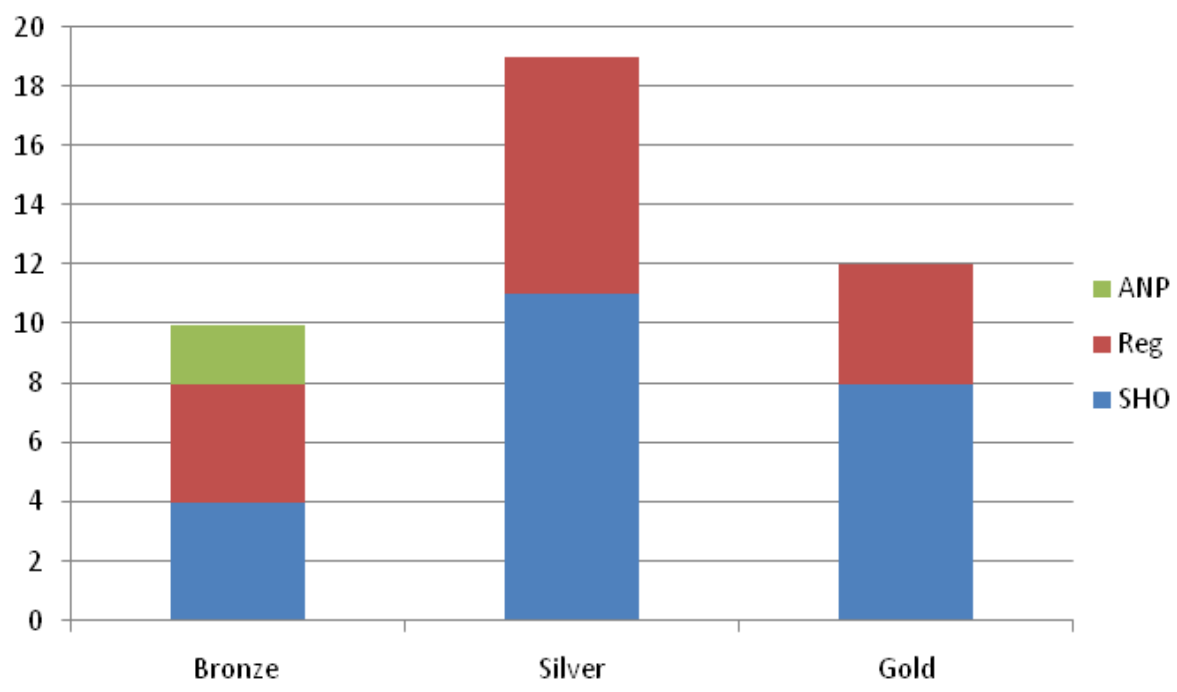

Graph I Scores per doctor grade during Cycle I. 


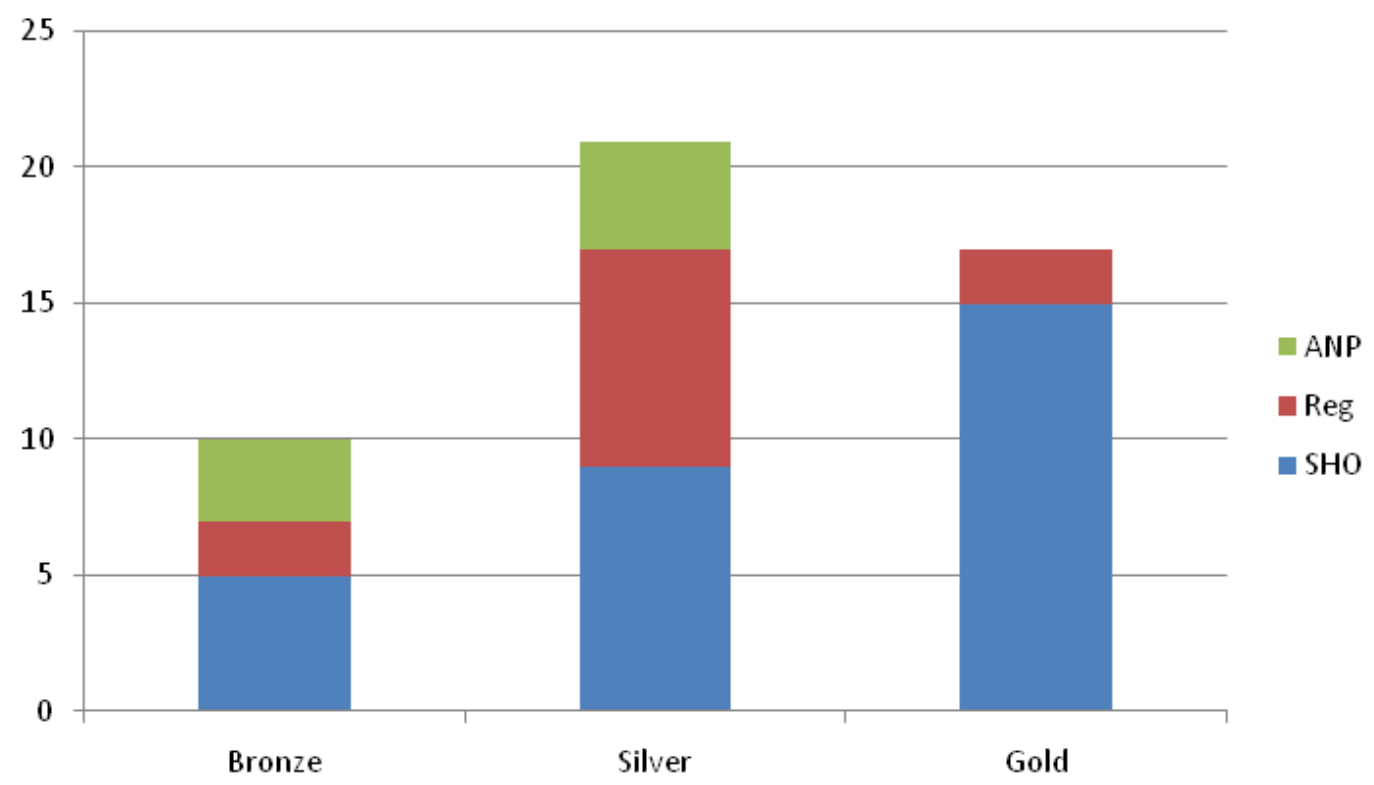

Graph 2 Scores per doctor grade during Cycle 2.

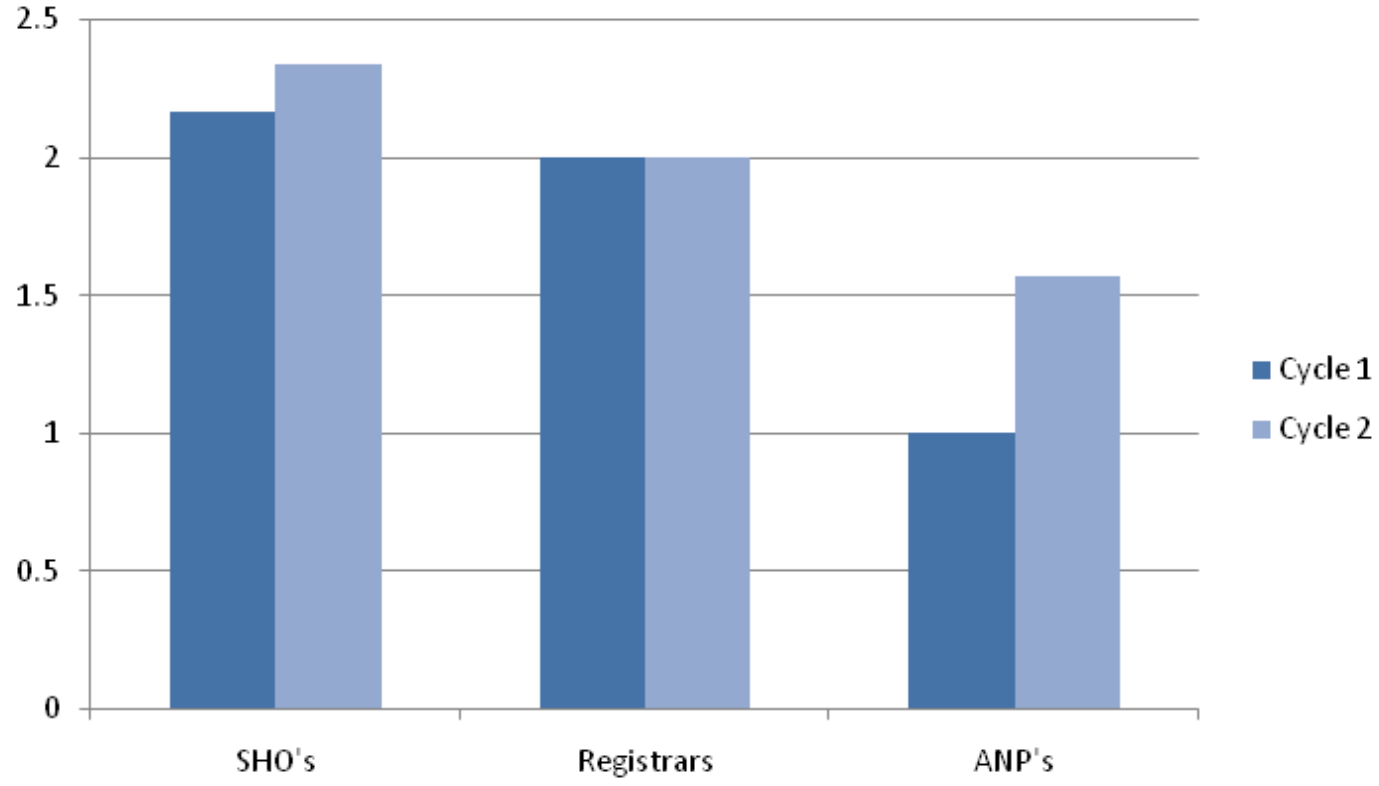

Graph 3 Improvement in score: SHO's vs. Registrar/Staff Grade.

\section{Discussion}

Simple education sufficed to close the gap towards best practice, especially for junior grade doctors and ANP's. During the process no serious pathology was missed. Revalidation and confidencebuilding for doctors in training achieved a higher consistency in clinical examination. The lack of confidence of non- ENT specialists in examining uncooperative children is potential factor, with a need for more robust undergraduate confidence-building in this skill, and/ or for it to be re-taught as part of a paediatric departmental induction. There is a need to develop tools for eliciting increased cooperation from children in this scenario. ${ }^{x}$ The scoring system used was a helpful feedback mechanism, articulating to doctors how their standards measured up against best practice and motivating improvement.

\section{Conclusion}

1. Full Ear, Throat and Neck examinations should be carried out for children presenting with feverish illness

2. Feedback to front line clinicians is helpful in maintaining best practice with the help of scoring systems

3. Further education and revalidation of these clinicians is called for in light of the challenges presented by children and COVID -19 , particularly for training grade, non ENT specialists. 


\section{Acknowledgments}

Thanks to Dr Fiona Hillis, Consultant Paediatrician, Royal Alexandra Hospital, for advice on the design and relevant guideline framework.

\section{Conflicts of interest}

No conflict of interest to declare.

\section{Endnotes}

i. Rationale and impact $\mid$ Fever in under 5s: assessment and initial management | Guidance | NICE. Nice.org.uk. https://www.nice.org.uk/ guidance/ng143/chapter/Rationale-and-impact\#kawasaki-disease-2. Published 2020. Accessed May 23, 2020.

ii. NHS Greater Glasgow and Clyde. Feverish Illness In Children $(<5$ Years) Paediatric Emergency Department, RHC. Glasgow; 2020.

iii. Bickely L. Bates' Guide To Physical Examination And History Taking. 7th ed. Philadelphia: Lippincot Williams and Wilkins; 1999 ch. 18.

iv. Demir E, Topal S, Atsal G, Erdil M, Coskun ZO, Dursun E. Otologic Findings Based on no Complaints in a Pediatric Examination. Int Arch Otorhinolaryngol. 2019;23(1):36-40. v. Wang E, Brar K. COVID-19 in children: an epidemiology study from China [published online ahead of print, 2020 Apr 21]. J Allergy Clin Immunol Pract. 2020.

vi. Evaluating and Testing Persons for Coronavirus Disease 2019 (COVID-19). Centers for Disease Control and Prevention. https://www. cdc.gov/coronavirus/2019-ncov/hcp/clinical-criteria.html. Published 2020. Accessed May 23, 2020.

vii. Franzese CB. Pilot study of an Objective Structured Clinical Examination ("the Six Pack") for evaluating clinical competencies. Otolaryngol Head Neck Surg. 2008;138(2):143-148.

viii. MAXIM B, DIELMAN T. Dimensionality, internal consistency and interrater reliability of clinical performance ratings. Med Educ. 1987;21(2):130-137.

ix. Sergeyev Y. The Olympic Medals Ranks, lexicographic ordering and numerical infinities. The Mathematical Intelligencer. 2020;37(2):4-8. https://arxiv.org/abs/1509.04313v1. Accessed May 23, 2020.

x. Leclerc JE, Julien AS, Leclerc LD, Bergeron-Dupras T. Tips for children's better cooperation during the examination of the head and neck: A Delphi method. Int J Pediatr Otorhinolaryngol. 2016;86:233-239. 